\title{
Economic Advantages of Office Buildings Providing Ancillary Services with Intraday Participation
}

\author{
Faran A. Qureshi, Ioannis Lymperopoulos, Ali Ahmadi Khatir, and Colin N. Jones
}

\begin{abstract}
Controlling the consumption profile of office buildings can be used to provide balancing services to the power grid at a financial benefit without violating its thermal comfort constraints. The economic advantage of such a service is a reduction in the total operating cost, but also an increase in average occupants' comfort when using our control scheme. We study these effects for the case of participating in the secondary frequency control market of Switzerland. Moreover, we examine the advantages of engaging in the intraday energy market. We propose a method for solving the flexibility bidding problem for a building in order to partake in the ancillary services market. The proposed solution is based on the combination of a new intraday control policy and two-stage stochastic programming. We also study the sensitivity of this economic benefit to electricity pricing. Our findings are based on extensive simulations with real data for energy prices, ancillary service bids, meteorological records and the frequency control signals for the year 2014 as transmitted by Swissgrid.
\end{abstract}

Index Terms-demand response, building control, secondary frequency control, smart grids, stochastic optimization, MPC.

\section{INTRODUCTION}

$\mathbf{P}$ OWER grid operators are required to balance electricity production and consumption to guarantee the stability of the power system and the quality of the power output. A special set of reserves and services is dedicated to this operation, known as Ancillary Services (AS). The heart of AS is the frequency control service that responds to power grid contingencies at various time scales [1]. The system operators procure such standby capacity from Ancillary Services Providers (ASP). Traditionally, those have been generating units, while today flexible loads or storage systems can act also as ASPs [2].

Most imbalances were up to recently due to the stochastic fluctuation of consumption (with the exception of generating contingencies). The growing participation of renewables in the energy generation mix is increasing further the requirements for fast responding AS [3]. Since this trend is expected to continue, efficient and economic dispatch of AS will be a crucial part of modern power systems.

In aggregate, office buildings are a significant part of the electrical consumption. Moreover, they are a load coupled to thermal capacity and usually equipped with a controllable Heating Ventilation and Air conditioning (HVAC) system. In addition, large office buildings are commonly equipped with

Authors are with the Automatic Control Laboratory, EPFL, Switzerland (email: faran.qureshi, ioannis.lymperopoulos, ali.khatir, colin.jones@epfl.ch).

This work has received support from the Swiss National Science Foundation under the GEMS project (grant number 200021 137985) and the European Research Council under the European Unions Seventh Framework Programme (FP/2007-2013) / ERC Grant Agreement n. 307608 (BuildNet). a central building management system which automates the control process of thermal comfort. The main target of the control effort is to maintain occupant comfort despite external effects (weather, occupancy, etc) at the minimum operational cost. We propose here that participation in the AS can further reduce the costs with a positive impact in average comfort.

Various studies have demonstrated the ability of buildings to shift their power consumption from peak to off-peak hours without sacrificing occupant comfort [4]. In [5], the authors propose a robust optimization based approach to evaluate the flexibility of a building HVAC system. A robust optimization based hierarchical control scheme was presented in [6] enabling an aggregation of commercial buildings providing also AS to the grid. [7] discussed a contract design problem for aggregators providing AS. Control of air handing units to track a regulation signal was demonstrated by [8]. [9], [10] presented experimental studies verifying a building's capability to provide ancillary service. A method based on robust optimization to characterize a building's consumption flexibility as a virtual battery, enabling it to offer it to the grid was presented in [11]. Most works focus on the technical capability of buildings providing AS, without assessing the economic feasibility or taking into account a realistic market structure, while the solution of the bidding problem does not consider participation on the intraday energy market. Finally, no analysis on how to optimize financial performance by incorporating all mechanisms (weekly bids, day-ahead auctions, intraday market) of the energy and AS market has been performed.

Participation in the intraday energy market gives the capacity to the building to change its contracted power consumption during the course of the day. By doing so, the building may effectively charge / discharge its stored energy. [12] proposed a method to charge / discharge the storage system (acting as an ASP) at a frequency slower than the tracking signal using intraday market, bilateral agreements, or pooling with a power plant such that a smaller storage size is required to provide frequency tracking services.

This paper is an extension of our previous work [13], where a preliminary economic analysis of an office building providing ancillary service (without participating in the intraday market) to the grid in Switzerland was presented. Here we refine these results by incorporating more realistic conditions. Moreover, an approximate solution method is proposed for the stochastic bidding problem (faced by the building providing ancillary services) with intraday market participation. We propose a methodology to design the intraday control policy separately before formulating the bidding problem. A simula- 
tion study is carried out evaluating the economic potential of an office building in Lausanne providing secondary frequency control service to Swissgrid, the Swiss Transmission System Operator (TSO). We study also the additional economic benefit of participating in the Swiss intraday market. Providing secondary frequency control to the grid results in savings for the building which are further increased by participating in the intraday market. Furthermore, we obtained the counterintuitive result of increased occupants' comfort at a reduced price for buildings providing secondary frequency control to the grid. This is because of the extra energy consumed to provide flexibility to the grid. Finally, we investigate the sensitivity of the economic advantages to the electricity price.

The swiss AS market structure is described in Section II] the problem formulation and modeling is presented in Section III. followed by the proposed approximate solution method in Section IV results and detailed simulation study is presented in Section V, and finally conclusions are drawn in Section VI.

Notation: Bold letters denote sequence of vectors over time, the length of which is clear from context, e.g., $\mathbf{e}=$ $\left[e_{0}^{T}, e_{1}^{T}, \ldots, e_{N-1}^{T}\right]^{T}$.

\section{SWISS ANCILlary SERVICES AND SPOT MARKET}

To maintain grid stability, the grid operator employs three different levels of control, namely, primary, secondary, and tertiary frequency control to achieve balance of production and consumption at various time scales $[1],[14]$. In this paper we focus on the secondary frequency control for Swissgrid.

\section{A. Secondary Frequency Control}

Swissgrid procures secondary control reserve capacity in a weekly auction from a set of pre-qualified ASPs. Swissgrid activates the acquired reserve capacity by sending a real-time activation signal to all eligible ASPs (the eligibility is decided in a weekly auction). The activation signal for each ASP is proportional to its accepted capacity and is a scaled version of the normalized activation signal which is called the automatic generation control (AGC) signal in Switzerland.

The ASP could be either a generator or a load, e.g., for surplus energy in the grid, a generator ASP reacts by decreasing its production or equivalently a load ASP reacts by increasing consumption. We focus only on loads here, and all references to ASP is from a load point of view. By convention, a positive AGC signal refers to loads consuming more and vice versa.

The ASPs participate in the Swiss secondary frequency control service in two phases (offline and online). During the offline phase an auction is conducted every week, where all ASPs declare their available flexibility $\gamma$, for the upcoming week, in reference to their nominal (baseline) power consumption $\overline{\mathbf{e}}$. The flexibility is offered as the maximum scaling of AGC signal a, the ASP will be able to track, at a specific price. The baseline consumption is declared either in the dayahead or intraday energy market. During the online phase, an AGC signal is propagated from Swissgrid, which all ASPs are required to track. Tracking the signal means that the difference between the total power consumption (on a second by second calculation) of the ASP and its baseline power consumption is close to the scaled version of the signal $\gamma \mathbf{a}$ according to requirements published by Swissgrid. Details of the costs, benefits and structure of these phases and the energy markets, are discussed below.

\section{B. Offline / Bidding Phase}

1) Weekly AS Capacity Auction: Every week, the ASP bids a certain capacity $\gamma \mathrm{MW}$ in the secondary frequency control market. This is the flexibility in terms of maximum deviation from the baseline power consumption that the ASP can offer to the grid, and in turn, receives a reward

$$
R_{\text {capacity }}(\gamma):=c_{\text {capacity }} \gamma
$$

where $c_{\text {capacity }}$ is the bid price of the ASP.

2) Day Ahead Auction: Each day, the ASP participates in the day-ahead spot market to buy the baseline power profile $\overline{\mathrm{e}} \mathrm{MWh}$ for each $15 \mathrm{~min}$ increment of the next day. Its cost is defined as

$$
C_{\text {baseline }}(\overline{\mathbf{e}}):=\mathbf{c}_{\text {electricity }}^{T} \overline{\mathbf{e}}
$$

where $\mathbf{c}_{\text {electricity }}$ is the electricity price which is the sum of the day-ahead spot market price, distribution charges, and taxes. $\mathbf{c}_{\text {electricity }}$ is unknown at the time of bidding the capacity $\gamma$. Note, that the distribution charges and taxes may differ depending on the physical location of the ASP within Switzerland and the level of the distribution network at which it is connected to the grid.

\section{Online Phase}

1) Intraday Transaction Cost: As the day progresses, the ASP can re-adjust its baseline power consumption in the intraday market. The ASP can buy or sell energy $\mathbf{m}$ MWh for any hour of the day, at least $75 \mathrm{~min}^{1}$ before the hour-ofinterest, from other market participants. This means that the ASP can still modify its predefined baseline power schedule. Participation in the intraday market is optional and the ASP may decide not to alter its baseline. The cost of intraday transactions is defined as

$$
C_{\text {intraday }}(\mathbf{m}):=\mathbf{c}_{\text {intraday }}^{T} \mathbf{m}
$$

where $\mathbf{m}$ is the intraday power and $\mathbf{c}_{\text {intraday }}$ is the intraday transaction price. Note that the intraday transaction price may vary with the time of purchase of the intraday power, however, we consider all intraday transactions to be exactly $75 \mathrm{~min}$ before the time-of-interest. $\mathbf{c}_{\text {intraday }}$ can be higher or lower compared to the electricity price $\mathbf{c}_{\text {electricity }}$. The intraday cost $C_{\text {intraday }}$ may be either positive or negative, depending on net buying or selling of energy.

Swissgrid remunerates the ASP by measuring its total power consumption $e_{i}$ every 15 min. Financial adjustments are made based on the 15 min measurements, and have two parts, (i): The AGC Tracking Reward - Incentivizing the price of energy consumed / produced by AGC tracking, and (ii): Tracking Error Penalty - penalizing the tracking errors.

\footnotetext{
${ }^{1}$ Minimum lead time of 75 min was applicable in 2014. Since 16 July 2015 this has been reduced to $60 \mathrm{~min}$.
} 
2) AGC Tracking Reward: If the received AGC signal is positive, the ASP increases its consumption. This extra energy is charged at a reduced price $\mathbf{c}_{A G C}$ as a bonus. Similarly, for tracking a negative AGC signal, the ASP decreases its consumption, and in turn, receives a rebate at a price $\mathbf{b}_{A G C}$ on unused energy. The total reward received by the ASP for tracking the AGC signal $\gamma \mathbf{a}$ is therefore given as

$$
R_{A G C}(\gamma, \mathbf{a}):=-\mathbf{c}_{A G C}^{T} \max \{\gamma \mathbf{a}, 0\}+\mathbf{b}_{A G C}^{T} \max \{-\gamma \mathbf{a}, 0\}
$$

where $\mathbf{c}_{A G C} \leq \mathbf{c}_{\text {electricity }} \leq \mathbf{b}_{A G C}$.

3) Tracking Error Penalty: The tracking service provided by the ASP, during the online tracking phase, is the difference between the total power consumption $\mathbf{e}$, and the net baseline schedule $\overline{\mathbf{e}}+\mathbf{m}$. The tracking error $\epsilon$ is defined as

$$
\epsilon=\mathbf{e}-\overline{\mathbf{e}}-\mathbf{m}-\gamma \mathbf{a}
$$

Swissgrid imposes penalties $C_{\text {penalty }}$ on $\epsilon$ to maintain tracking quality. Different penalties are paid for positive and negative tracking error and the total penalty is given as

$$
C_{\text {penalty }}(\epsilon):=\mathbf{c}_{\text {penalty }}^{T} \max \{\epsilon, 0\}-\mathbf{b}_{\text {penalty }}^{T} \max \{-\epsilon, 0\}
$$

where $\mathbf{c}_{\text {penalty }}$ is the cost, and $\mathbf{b}_{\text {penalty }}$ is the rebate paid for tracking errors. Readers are referred to [15] for more details of the Swiss AS market.

Finally, the Swissgrid regulations require the tracking errors to be smaller than a predefined ratio $q$ of the offered capacity $\gamma$, resulting in the following linear constraint

$$
\|\mathbf{e}-\overline{\mathbf{e}}-\mathbf{m}-\gamma \mathbf{a}\|_{\infty} \leq q \gamma
$$

\section{Problem Formulation}

In this section we formulate the bidding problem for participating in the secondary frequency control service of Swissgrid.

\section{A. Building Thermodynamics}

A state space model of the building thermodynamics is extracted from an EnergyPlus model, using the MATLAB toolbox OpenBuild [16]. EnergyPlus models are high fidelity, but due to their complexity, are not fit for optimization. The OpenBuild toolbox extracts all relevant data and constructs a linear continuous-time state space model of the thermodynamics. The procedure used is based on first principles modeling. The physical phenomena incorporated in OpenBuild include heat transfer through conduction, convection, long-wave radiation, and short-wave radiation. The impact of external weather conditions (outside temperature, solar gain, etc.), and internal gains (heat transfer due to occupants, electrical equipment, and lights) is also added to the model as a disturbance input. The quality of the building models obtained from OpenBuild have been validated through a comparison study with the original high fidelity EnergyPlus models. See [16] for further details.

The continuous-time linear model obtained from OpenBuild is approximated using the standard Hankel-Norm model reduction method and discretized. Finally, we obtain a model of the following form:

$$
\begin{aligned}
x_{i+1} & =A x_{i}+B_{u} u_{i}+B_{d} d_{i} \\
y_{i} & =C x_{i}
\end{aligned}
$$

where $x_{i} \in \mathbb{R}^{n}$ is the state, $u_{i} \in \mathbb{R}^{m}$ is the thermal power input to each zone of the building, $d_{i} \in \mathbb{R}^{p}$ is the disturbance input (outside temperature, solar gain, internal gains, etc.), and $y_{i} \in \mathbb{R}^{q}$ is the temperature in each zone at time step $i$.

The temperature in each zone influences occupants comfort, and therefore it is controlled to stay within certain acceptable bounds. This is incorporated as a linear constraint on the output of the system. We define the comfort constraint of level $\theta_{i}$, at time step $i$, by $\left|y_{i}-T_{\text {ref }}\right| \leq \theta_{i}$, where $T_{\text {ref }}$ is the optimal zone temperature, and $\theta_{i}$ is the deviation from the optimal temperature. The thermal input to each zone of the building is constrained by the physical limits of the HVAC system, which translates into an input constraint.

We define the set of admissible thermal power trajectories as the set of all the possible thermal power inputs that the building can consume, over a horizon $N$, while meeting the comfort requirements and actuator limitations, described as

$$
\mathcal{U}(x)=\left\{\mathbf{u} \mid \begin{array}{l}
x_{i+1}=A x_{i}+B_{u} u_{i}+B_{d} d_{i} \\
\left|C x_{i}-T_{\mathrm{ref}}\right| \leq \theta_{i} \\
u_{i} \in \mathbb{U} \\
x_{0}=x, \quad \forall i=0, \ldots, N-1 .
\end{array}\right\}
$$

where $x$ is the initial state of the building and $\mathbb{U}$ is the set defining the actuator limits.

\section{B. HVAC System and Thermal Storage}

Thermal storage constitutes an integral part of modern HVAC systems. Storage systems are installed for two main reasons. Firstly, to reduce the operational cost by shifting electrical power consumption from expensive peak hours to cheaper off-peak hours. Secondly, to reduce the size of the heating / cooling system required to meet the peak thermal load. A generic thermal storage model takes the following form

$$
s_{i+1}=\alpha s_{i}+\beta_{\text {in }} e_{i}-\beta_{\text {out }} p_{i}
$$

where $s_{i} \in \mathbb{R}$ is the state of the storage, $e_{i} \in \mathbb{R}$ is the electrical power consumed, and $p_{i} \in \mathbb{R}$ is the thermal power out of the storage at time step $i$. Moreover, $\alpha$ is the dissipation rate of the storage, $\beta_{i n}$ is the coefficient of performance (COP) of the HVAC system, and $\beta_{\text {out }}$ is thermal power loss in discharging the storage. The COP of the heating / cooling system is defined as the net efficiency of converting electrical power to thermal power.

The storage state is constrained by the physical size of the storage $\left(s_{i} \in \mathbb{S}\right)$, and the electrical power input is constrained by the power rating of the installed (heating / cooling) equipment $\left(e_{i} \in \mathbb{E}\right)$. We define the set of all the possible electrical and thermal power consumption trajectories, over a horizon $N$, as the set of admissible electrical and thermal power which is given as

$$
\mathcal{S}(s)=\left\{\begin{array}{l|l}
(\mathbf{e}, \mathbf{p}) & \begin{array}{l}
s_{i+1}=\alpha s_{i}+\beta_{\text {in }} e_{i}-\beta_{\text {out }} p_{i} \\
s_{i} \in \mathbb{S} \\
e_{i} \in \mathbb{E} \\
s_{0}=s, \quad \forall i=0, \ldots, N-1 .
\end{array}
\end{array}\right\}
$$


Thermal storage is assumed to be in parallel operation with the building and at the output of the HVAC. Thus, the electrical heating / cooling system can either provide the thermal power to the building directly, or charge the thermal storage. On the other hand, the building can use both the storage and the heating / cooling system to meet its thermal load. This implies that the total thermal power consumed by the building is equal to the thermal power output of the storage and is expressed as the following linear constraint

$$
\mathbf{p}=\Gamma \mathbf{u}
$$

where $\Gamma:=I_{N} \otimes \mathbf{1}^{T}$, with $I_{N}$ an identity matrix of size $N$, and $\otimes$ is the Kronecker product.

\section{Bidding Problem}

The objective of the bidding problem is to select the capacity bid $\gamma$, and the baseline power consumption for the first day $\overline{\mathbf{e}}$, which minimizes the expected operational cost, while maintaining occupant comfort with high probability. The total cost of operation is the sum of all the costs and rewards introduced in Section II, while the operational constraints are expressed by (7), (9), (11), and (12). We formulate the bidding problem as the following optimization problem

$$
\begin{array}{cl}
\underset{\gamma, \pi_{\bar{e}}, \pi_{e}, \pi_{m}, \pi_{u}}{\operatorname{minimize}} & C_{\text {baseline }}(\overline{\mathbf{e}})-R_{\text {capacity }}(\gamma) \\
& +\mathbb{E}_{\mathbf{a}}\left[C_{\text {penalty }}(\epsilon)-R_{A G C}(\gamma, \mathbf{a})\right. \\
& \left.+C_{\text {intraday }}(\mathbf{m})\right] \\
\text { s.t. } & \mathbf{u} \in \mathcal{U} \\
& (\mathbf{e}, \mathbf{p}) \in \mathcal{S} \\
& \mathbf{p}=\Gamma \mathbf{u} \\
& \|\mathbf{e}-\overline{\mathbf{e}}-\mathbf{m}-\gamma \mathbf{a}\|_{\infty} \leq q \gamma \\
& \gamma \geq 0 \\
& \overline{\mathbf{e}}=\pi_{\bar{e}}(\mathbf{a}), \quad \mathbf{e}=\pi_{e}(\mathbf{a}) \\
& \mathbf{u}=\pi_{u}(\mathbf{a}), \quad \mathbf{m}=\pi_{m}(\mathbf{a})
\end{array}
$$

where the AGC signal a is uncertain, and the decision variables are the baseline power consumption $\overline{\mathbf{e}}$, the capacity bid $\gamma$, the total electrical power consumption e, the intraday transaction $\mathbf{m}$, and the the thermal power consumption of the building $\mathbf{u}$. Since the final cost depends on the realization of the AGC signal, we take its expectation over a. Typically, the comfort constraints are formulated as chance constraints while the operational and tracking constraints are handled in a robust fashion.

At the time of the decision, the AGC signal is unknown, therefore $(13)$ is a multi-stage uncertain optimization problem [17]. The problem uncertainty is revealed at different stages, and it is possible to re-adjust the control action accordingly. The capacity $\gamma$ and the baseline for the first day $\overline{\mathbf{e}}$ are the first stage variables, while $\mathbf{e}, \mathbf{m}, \mathbf{u}$, and baseline for the rest of the week $\overline{\mathbf{e}}$ are the subsequent stage variables. Therefore, for subsequent stage variables, the goal is to optimize over the control policies $\overline{\mathbf{e}}=\pi_{\bar{e}}(\mathbf{a}), \mathbf{e}=\pi_{e}(\mathbf{a}), \mathbf{u}=\pi_{u}(\mathbf{a}), \mathbf{m}=$ $\pi_{m}(\mathbf{a})$, rather than a fixed trajectory over the whole horizon. Furthermore, the policies must be causal, i.e., the decision at time step $i$ depends only on the realization of uncertainty until time $i$.
Multi-stage uncertain optimization problems are known to be intractable [17], thus obtaining an exact solution of the bidding problem (13) can become challenging especially for long time horizons.

\section{Approximate Solution Method}

In this section we present an approximate solution method for the bidding problem (13). The key idea is to separate the intraday control policy from the bidding problem and to solve it independently. We first present the proposed causal intraday control policy which is a function of previously received AGC signal a. Next, the bidding problem is approximated by a twostage stochastic optimization problem using the pre-defined intraday policy.

\section{A. Intraday Control}

The AGC mean might exhibit a considerable bias in either direction for short time horizons. This creates an amount of extra energy consumed or removed from the building compared to its baseline (nominal) consumption.

The building may decide to participate in the intraday market to counteract such temporal deviations of AGC from zero mean. This implies that the building may re-adjust its baseline (declared) power consumption, depending on its current state and the net energy deviation derived from AGC. For example, if the cumulative sum of the AGC signal on a certain day is positive, i.e., the building consumed extra energy to track the AGC, then, it may compensate for that by reducing its future baseline consumption. Thus, by participating in the intraday market, the building may effectively offer a higher tracking capacity to the grid for the same physical storage size.

We define the residual tracking signal $\mathbf{r}$ as the sum of the received $\mathrm{AGC}$ and the intraday transaction, i.e., the signal required to be tracked by the building after making the intraday adjustments to the baseline power consumption. The normalized residual tracking signal is given as

$$
\mathbf{r}=\mathbf{a}+\overline{\mathbf{m}}
$$

where $\overline{\mathbf{m}}$ is the normalized intraday transaction. $\overline{\mathbf{m}}$ denotes the intraday transaction corresponding to the received normalized AGC signal a. Total intraday transaction $\mathbf{m}=\gamma \overline{\mathbf{m}}$ is a scaled version of $\overline{\mathbf{m}}$.

We here propose a causal control policy of intraday transaction $\overline{\mathbf{m}}=\pi_{m}(\mathbf{a})$, such that the residual tracking signal has a smaller bias (cumulative sum) over the horizon, and a mean closer to zero. The cumulative sum of a signal $\mathbf{a}$, from time step $j$ to $k$ is defined as $\hat{a}_{j}^{k}=\sum_{i=j}^{k} a_{i}$. The intraday control policy is then

$$
\pi_{\overline{\mathbf{m}}}(\mathbf{a})=\left\{\overline{\mathbf{m}} \mid \begin{array}{ll}
\bar{m}_{i+1}= & \\
\begin{array}{l}
\operatorname{mamin}_{i+1} \\
\bar{m}_{0}+\bar{m}_{i+1}+\mathbb{E}_{a}\left[\hat{a}_{i}^{i+1}\right] \\
\text { s.t. }
\end{array} & \hat{r}_{0}^{i}=\hat{r}_{0}^{i-1}+\bar{m}_{i}+\hat{a}_{i-1}^{i} \\
& \hat{r}_{0}^{-1}=0, \bar{m}_{0}=0, \hat{a}_{-1}^{0}=0 \\
& \forall i=0, \ldots, N-1 .
\end{array}\right\}
$$

where $\bar{m}_{i}$ is the normalized intraday action at time step $i, \hat{r}_{0}^{i}$ is the cumulative sum of the residual tracking signal from time 
step 0 to $i$, and $\hat{a}_{i}^{i+1}$ is the cumulative sum of the AGC signal received between time step $i$ and $i+1$. Note that $(15)$ is a causal multi-stage control policy. At time step $i$, the control policy (15) measures the cumulative sum of the received AGC signal in the interval $i-1$ to $i$ and updates the cumulative sum of the residual tracking signal $\hat{r}_{0}^{i}$ using the previously optimized intraday action $\bar{m}_{i}$. Then, the intraday action for the next time step $\bar{m}_{i+1}$ is optimized by minimizing the expected value of the cumulative sum of the residual tracking signal at the next time step. The AGC signal at the next time step is not yet realized and is uncertain. This procedure is repeated recursively, as the uncertainty is revealed. The expected value can be estimated at each step using scenarios of the AGC signal.

Remark 1. The actual tracking signal is a scaled version of a. Similarly, the actual intraday transaction is $\mathbf{m}=\gamma \overline{\mathbf{m}}$, and the resulting residual tracking signal required to be tracked by the building after making intraday adjustments is a scaled version of $\mathbf{r}$.

Remark 2. The intraday transaction will incur a cost $C_{\text {intraday }}(\mathbf{m})=\mathbf{c}_{\text {intraday }}^{T} \gamma \overline{\mathbf{m}}$ as already defined in Section II. Note that this part of the total cost in the bidding problem is now a function of $\gamma$ only, since $\overline{\mathbf{m}}$ is already fixed with the intraday policy (15).

Remark 3. Note that given the scenarios of the normalized AGC signal over the horizon $\mathbf{a}^{j}$, the control policy (15) can be used to obtain the resulting scenarios of the intraday action $\overline{\mathbf{m}}^{j}$, and the residual tracking signal $\mathbf{r}^{j}$.

\section{B. Two-stage Stochastic Approximation}

To approximate the bidding problem we reduce the multistage structure of the optimization to two stages. The causality requirements are relaxed, and it is assumed that after the first stage variables are selected, the uncertainty is revealed over the whole horizon in the second stage, and it is possible to readjust the second stage control actions after the uncertainty is realized. The first stage variables are $\gamma$, and $\overline{\mathbf{e}}$ over the whole week, while $\mathbf{e}$ and $\mathbf{u}$ are the second stage variables. Instead of the multi-stage policy $\mathbf{e}=\pi_{e}(\mathbf{a})$, and $\mathbf{u}=\pi_{u}(\mathbf{a})$, a twostage control policy is used for the second stage variables. The intraday control policy $\mathbf{m}=\pi_{m}(\mathbf{a})$ is already fixed, and is not an optimization variable anymore. Moreover, the received AGC signal is transformed to the residual tracking signal $\mathbf{r}$ using (14).

Once the capacity $\gamma$ and the baseline power consumption $\overline{\mathbf{e}}$ are fixed in the first stage (the intaday transaction $\mathbf{m}$ is also fixed), the best strategy is to minimize the tracking error penalty $C_{\text {penalty }}$, subject to the comfort requirements and the operational constraints. We define $C_{\text {penalty }}^{*}$ as the optimal value of the tracking penalty, given that the first stage variables are fixed and the operational constraints are satisfied

$$
\begin{array}{ll}
C_{\text {penalty }}^{*}(\psi, \phi):=\underset{\mathbf{e}, \mathbf{u}}{\operatorname{minimize}} & C_{\text {penalty }}(\mathbf{e}-\psi) \\
\text { s.t. } & \mathbf{u} \in \mathcal{U} \\
& (\mathbf{e}, \mathbf{p}) \in \mathcal{S} \\
& \mathbf{p}=\Gamma \mathbf{u} \\
& \|\mathbf{e}-\psi\|_{\infty} \leq q \phi
\end{array}
$$

The approximate bidding problem is given as

$$
\begin{array}{ll}
\underset{\overline{\mathbf{e}}, \gamma}{\operatorname{minimize}} & C_{\text {baseline }}(\overline{\mathbf{e}})-R_{\text {capacity }}(\gamma) \\
& +\mathbb{E}_{\mathbf{a}}\left[C_{\text {penalty }}^{*}(\overline{\mathbf{e}}+\gamma \mathbf{r}, \gamma)\right. \\
& \left.-R_{A G C}(\gamma, \mathbf{a})+C_{\text {intraday }}(\gamma \overline{\mathbf{m}})\right] \\
\text { s.t. } & \gamma \geq 0
\end{array}
$$

where $\mathbf{r}$ is the normalized residual tracking signal defined by (14), and $\overline{\mathbf{m}}$ is the normalized intraday transaction defined by (15). The optimizer of (17) is the AGC tracking capacity $\gamma$ and the baseline power consumption $\overline{\mathbf{e}}$ for the whole week.

The two-stage stochastic optimization problem (17) can be solved using the well known sample averaged approximation method [17], where an implicit policy of the second stage decision variables is defined by having separate trajectories of the second-stage decision variables corresponding to each sample of the uncertain variable a, resulting in the following optimization problem

$$
\begin{array}{cl}
\underset{\overline{\mathbf{e}}, \gamma}{\operatorname{minimize}} & C_{\text {baseline }}(\overline{\mathbf{e}})-R_{\text {capacity }}(\gamma) \\
& +\frac{1}{N_{s}} \sum_{j=1}^{N_{s}}\left[C_{\text {penalty }}\left(\mathbf{e}^{j}-\overline{\mathbf{e}}-\gamma \mathbf{r}^{j}\right)\right. \\
& \left.-R_{A G C}\left(\gamma, \mathbf{a}^{j}\right)+C_{\text {intraday }}\left(\gamma \overline{\mathbf{m}}^{j}\right)\right] \\
\text { s.t. } \quad & \mathbf{u}^{j} \in \mathcal{U} \\
& \left(\mathbf{e}^{j}, \mathbf{p}^{j}\right) \in \mathcal{S} \\
& \mathbf{p}^{j}=\Gamma \mathbf{u}^{j} \\
& \left\|\mathbf{e}^{j}-\overline{\mathbf{e}}-\gamma \mathbf{r}^{j}\right\|_{\infty} \leq q \gamma \\
& \gamma \geq 0, \quad \forall j=1, \ldots, N_{s}
\end{array}
$$

where $N_{s}$ is the number of samples of the normalized AGC signal $\mathbf{a}$ and the corresponding samples of the normalized residual tracking signal $\mathbf{r}$. The superscript $j$ defines the second-stage decision variable corresponding to the $j^{\text {th }}$ scenario of the uncertain parameter. Note, that for each sample of the uncertain parameter $\mathbf{a}^{j}$ there are separate trajectories of the second stage optimization variables $\mathbf{e}^{j}$ and $\mathbf{u}^{j}$, also implicitly defining separate trajectories for $\mathbf{p}^{j}$ and the state variables.

Note that the original bidding problem (13) is a multi-stage uncertain optimization problem and is known to be intractable [17], thus obtaining an exact solution is challenging. Problem (16) is a linear program and (17) is the two-stage stochastic optimization problem defining the approximate solution of the bidding problem (13). Problem (18) is the scenario based tractable solution of the two-stage stochastic optimization problem (17), and can easily be transformed into a linear programming problem, and thus large scale problems can be solved efficiently using standard software tools.

\section{Simulation Analysis}

This section presents the simulation study of the optimization procedure and an analysis of the results. We define the following simulation cases:

Minimum Cost - No AGC tracking: The building minimizes its total energy cost of operation without participating in the AS market. We employ for this case a minimum cost MPC controller which has been widely studied in previous literature. AGC Tracking - No Intraday: The building minimizes its total cost of operation while providing the AGC tracking service, without participation in the Swiss intraday market. 


\begin{tabular}{ll}
\hline Building & \\
\hline Floor Area $\left(\mathrm{m}^{2}\right)$ & 511 \\
No. of Zones & 5 \\
Peak Occupancy (people $\left./ 100 \mathrm{~m}^{2}\right)$ & 5.4 \\
Maximum thermal power input (per zone) & $3.6 \mathrm{~kW}$ \\
\hline Thermal Storage & 1 \\
\hline Dissipation rate $\alpha$ & 2.4 \\
Average COP $\beta_{\text {in }}$ & $7.5 \mathrm{~kW}$ \\
Maximum electrical power consumption & \\
of the heating / cooling system & $150 \mathrm{kWh}$ \\
Maximum thermal energy capacity & $8.3 \mathrm{~h}$ \\
Full charge / discharge time & \\
\hline Comfort & $23^{\circ} \mathrm{C}$ \\
\hline Optimum ALD comfort temperature $T_{r e f}$ & $2{ }^{\circ} \mathrm{C}$ \\
Temperature variation (office hours) \\
Temperature variation (excl. office hours) & $4{ }^{\circ} \mathrm{C}$ \\
\hline
\end{tabular}

TABLE I: Building and Simulation Parameters

AGC Tracking - Intraday: The building minimizes the total cost of operation while providing the AGC tracking services to the grid and participating in the intraday energy market.

For comparison, all the above cases are repeated with and without a thermal storage tank in the HVAC system. The base case is taken to be the minimum cost operation without thermal storage tank.

\section{A. Simulation Setup}

The building employed in all simulations is the ASHRAE standard EnergyPlus model of a five zone office from the reference database of the U.S. department of Energy [18]. The building model is provided with typical usage patterns of electrical equipment, lights and occupancy schedule. The heating / cooling system and the thermal storage tank is sized using EnergyPlus. We consider an ideal thermal storage. For winter, this represents a hot water storage tank, while for summer an ice storage system. Main parameters are given in Table [. The assumptions of our simulations are listed below:

- Simulations are carried out for winter (weeks 2 to 10 , and 45 to 52) and summer (weeks 24 to 35) 2014.

- Recorded weather data of Lausanne for 2014 are used.

- Real energy prices for Lausanne for 2014 are used. We obtain the spot $\left(\mathbf{c}_{\text {electricity }}\right)$ and intraday index price $\left(\mathbf{c}_{\text {intraday }}\right)$ of electricity from the European Power Exchange (EPEX) [19].

- The average weekly capacity price $(\gamma)$, AGC tracking bonus $\left(\mathbf{c}_{A G C}, \mathbf{b}_{A G C}\right)$ and deviation penalty ( $\mathbf{c}_{\text {penalty }}$, $\mathbf{b}_{\text {penalty }}$ ) for the year 2014 are obtained from Swissgrid.

- We assume that the intraday market is liquid at all times.

- The historic normalized AGC signal (obtained from Swissgrid) is split into weekly signals which are used as scenarios. We draw $N_{s}=45$ random scenarios of the weekly AGC signal to solve the two stage program (18). $N_{s}$ is limited by the computational complexity of the resulting optimization problem, however, sensitivity studies suggest that the number is still representative of the underlying probability distribution.
- The AGC signal of the year 2014 is obtained from Swissgrid and is used in our simulations.

Remark 4. Building thermodynamics are slow, and are modeled with a sampling time of 15 minutes. From comfort pointof-view applying a fast thermal input signal is equivalent to applying a 15 minutes average of the fast signal. Moreover, most commercial HVAC systems (except electric heaters, and fans) cannot be controlled at rates faster than 15 minutes. Furthermore, all financial remuneration is cleared by Swissgrid using the 15 minutes average signals. Therefore, we use 15 minutes average data (including AGC signal) in our simulations. A device with fast dynamics (e.g. electric battery) will be required in practice to alter the consumption with high frequency. The size of the electric battery required to support AGC tracking at high frequency is determined by the worst-case energy content and power of the difference between the AGC signal received every second and the 15 minutes average AGC. This worst-case is estimated using historic $A G C$ scenarios and the analysis suggests that only a small supporting electric battery with power limit of $\pm 1 \mathrm{~kW}$, and a capacity of $0.04 \mathrm{kWh}$ is required when following the remaining of the AGC building tracked signal of $\pm 1 \mathrm{~kW}$ at a frequency of $1 s$.

1) Computations: The simulations are performed in MATLAB. The two-stage stochastic optimization problem (18) is formulated as a linear program using the YALMIP [20] toolbox for mathematical modeling and is solved using the Gurobi solver. It takes 40 minutes on average to solve the weekly bidding problem.

2) Solution Quality: The dispersion of the optimal cost is numerically estimated to evaluate the solution quality. Approximate two-stage stochastic program (18) is solved 20 times (for each case) for week-3 using different set of randomly drawn AGC scenarios. The estimated coefficient of variation (ratio of standard deviation to expected value) of the optimal cost is 0.028 , and 0.0122 for AGC Tracking with, and without intraday participation and without additional storage, while it is 0.0019 , and 0.0208 for the case of additional storage.

Small value of the coefficient of variation show that the dispersion of the optimal cost is small, and that the solution of $(18)$ is a reasonable estimate of the stochastic programming problem.

3) Comfort Measure: We use the ASHRAE Likelihood of dissatisfied (ALD) as a measure of occupants' comfort. ALD is a function of the deviation of zone temperature from the ideal temperature. ALD calculation is as a post-processing step and not a part of the optimization problem. Long-term percentage of dissatisfied (LPD) which is a function of ALD and the occupancy rate is used to evaluate the average comfort per week (for more details see [21]).

4) Simulation Run: The bidding problem (18) is solved with a horizon of one week (as required by Swissgrid), yielding the weekly capacity bid $\gamma$ and the baseline power consumption $\overline{\mathbf{e}}$. As the AGC signal arrives for the concerned week, in the real time phase, it defines the total power consumption of the building, i.e., the sum of the baseline power consumption and the scaled version of the received 

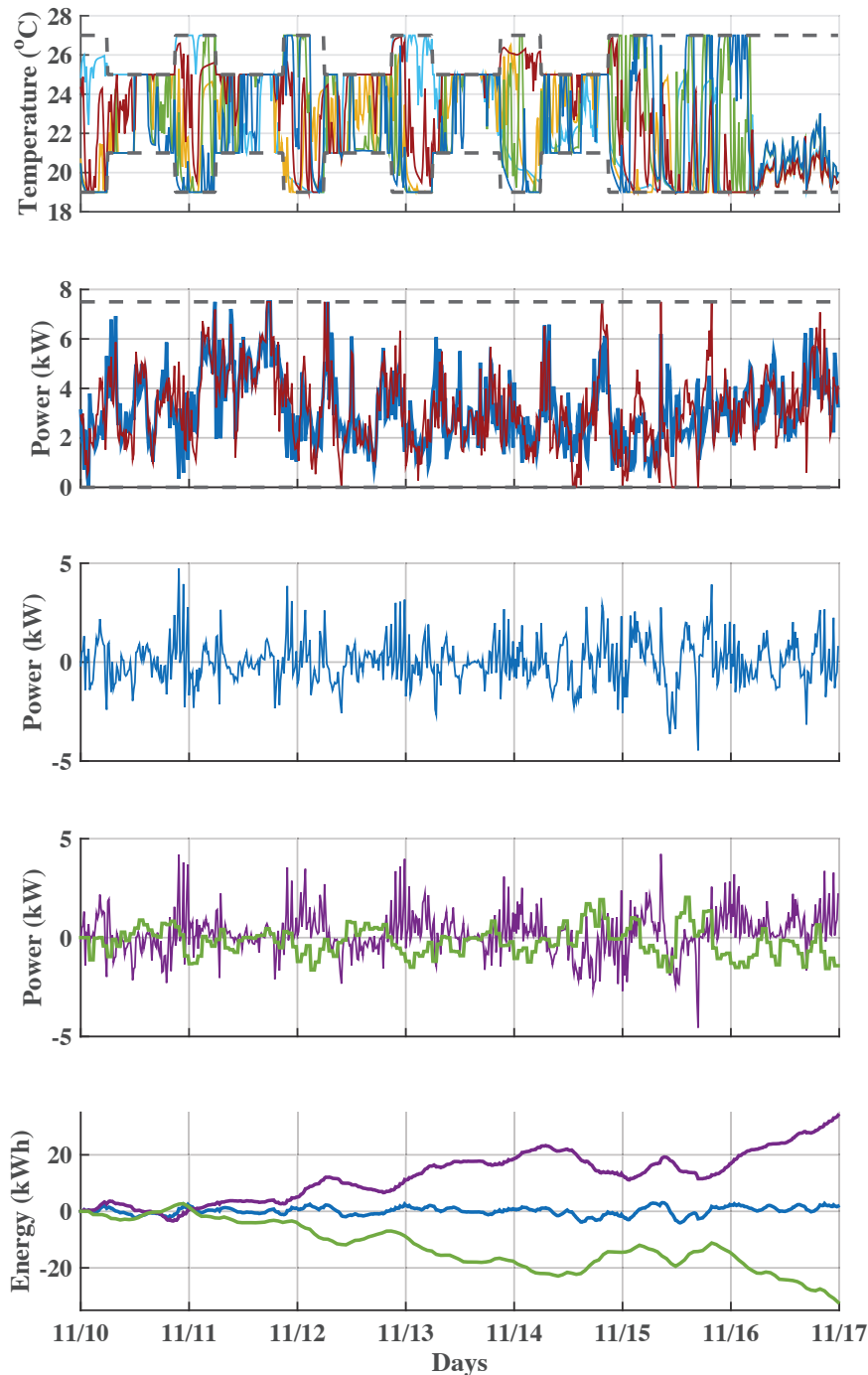

Fig. 1: Simulation result for week 46 (2014). Upper: Zone temperatures. Middle Up: Baseline power consumption $\overline{\mathbf{e}}$ (blue), total power consumption e (red). Middle: Residual tracking signal $\gamma \mathbf{r}$. Middle down: Received AGC signal $\gamma \mathbf{a}$ (purple), intraday transaction $\mathbf{m}$ (green). Lower: Cumulative sum of AGC (purple) / residual tracking signal (blue) / intraday transaction (green).

AGC signal. An open loop optimization problem determines the optimal distribution of thermal power in each zone of the building, while respecting the operational constraints. This gives us the resulting state trajectories. For the case where the building participates in the intraday market, the control policy (15) is used to obtain the intraday actions as described in Section IV]

The simulation result for week 46 is shown in Fig. 1 to demonstrate the effect of the proposed methodology. The received AGC signal is tracked while the zone temperatures stay within constraints. The received AGC signal, the residual tracking signal, the intraday transaction and their respective cumulative sum are also shown in Fig. 11. It can be seen that the causal intraday control policy (15) is effective in limiting

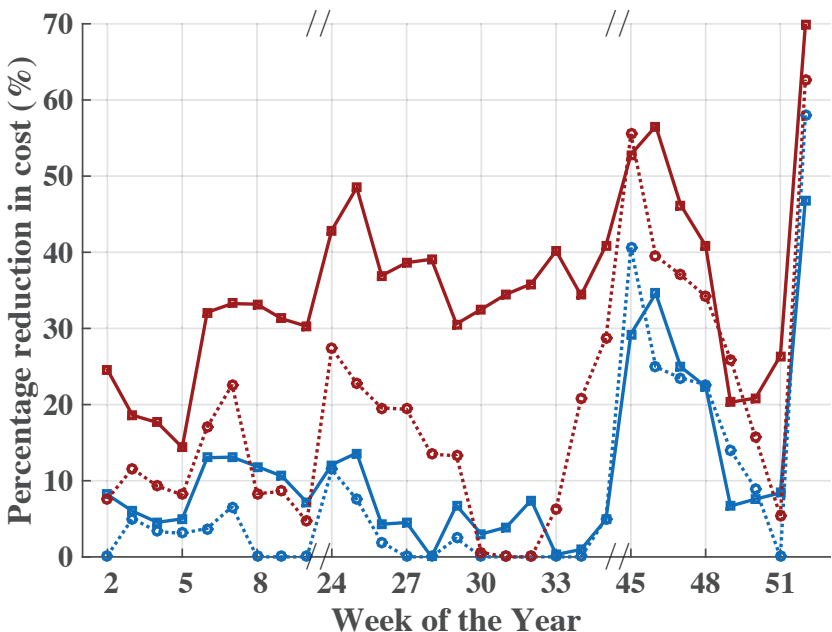

Fig. 2: Percentage reduction in weekly operation cost compared to the base case of minimum cost without additional thermal storage. Solid line: AGC Tracking - Intraday case, Dotted line: AGC tracking - No Intraday case. Red: Additional thermal storage, Blue: No additional thermal storage.

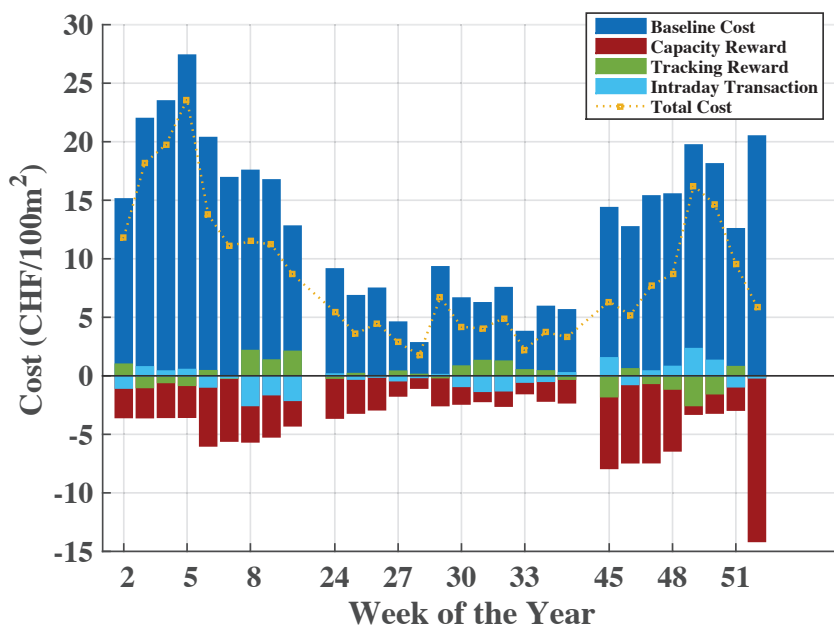

Fig. 3: Cost components for AGC tracking - Intraday with additional storage.

the cumulative sum of the residual tracking signal.

Comprehensive simulation results are presented next to study the financial aspect and benefit of participating in the Swiss AS program.

\section{B. Analysis of results}

Economic Benefit: The building participating in the Swiss AS, can reduce on average $18.8 \%$ its operational costs, while participating in the intraday energy market reduced them to $35.3 \%$. A building without extra storage saves on average $8.3 \%$ without, and $11.1 \%$ with intraday market participation. The percentage reduction in operational cost for all cases is depicted in Fig. 2. The percentage savings vary every week depending on the outside weather condition, electricity price, etc. 
On average, participating in the intraday market is advantageous for the building (solid line is above dotted line for most of the weeks). However, it is important to note that on any specific week, e.g., week 45 , the saving may be reduced by participating in the intraday market. This is because the intraday transaction cost for any specific week might be larger than the benefit of having a residual tracking signal with low energy.

Investing in the thermal storage system might be profitable under certain conditions. Comparing the total cost of operation when providing the ancillary service to the grid, with and without thermal storage, suggests that having thermal storage reduces the operating cost of the building on average $27.5 \%$ with, and $12.4 \%$ without intraday market participation.

Figure 3 illustrates the various components of the operational cost. The two most important parts are the baseline cost and the capacity bonus. Both, the tracking bonus and the intraday transaction cost may be positive or negative depending on the received AGC signal. The tracking error penalty is negligible, and not shown in this figure.

Impact of AGC tracking on occupant's comfort: The occupants' comfort is increased at a reduced cost (which is counter-intuitive). This is mainly due to the extra energy consumed to increase the baseline to provide (upwards and downwards) flexibility. In other words, the minimum price MPC aims at maintaining the temperature trajectories close to the constraints, while the zone temperatures are excited within the comfort limits when the building is participating in AGC tracking service, resulting in improved comfort.

To generate Fig. 4 we gradually tighten the comfort constraints toward the ideal zone temperature, and re-run the simulations for all cases (for week 4 and 29). Each of these simulations gives us a point on the price vs comfort axis, as shown in Fig. 4. For AGC tracking, the same comfort is achieved at a lower cost. The AGC tracking case with the presence of additional storage enables the building to attain best comfort at least price when it also participates in the intraday market. Similar trend is observed for summer and winter weeks.

Sensitivity to price of electricity: The economic benefits are sensitive to the electricity price. For a high electricity price, it might not be worth consuming extra energy, to increase the baseline for providing the AGC tracking capacity. As outlined in Section III the electricity price is the sum of the spot electricity price, the distribution charges, and taxes. The distribution charges vary within Switzerland depending on the physical location of the load. The analysis presented so far is for Lausanne with a distribution price of $100 \mathrm{CHF} / \mathrm{MWh}$.

We study the impact of varying the distribution price between (40 CHF/MWh and $160 \mathrm{CHF} / \mathrm{MWh}$ ) the range seen across Switzerland. Results are depicted in Fig. 5. The percentage reduction in operating cost increases with a decrease in the distribution charge. Furthermore, for the case of AGC tracking - No intraday and without additional storage, it is not worth providing the tracking service to the grid for a distribution price above $140 \mathrm{CHF} / \mathrm{MWh}$. However, participation in the intraday market still makes AGC tracking worthwhile for this case.
Office buildings in locations with lower distribution prices can benefit more from providing AS to the grid.

\section{CONCLUSION}

We presented an approximate solution method to the stochastic bidding problem with the possibility of participating in the intraday energy market. The simulation results demonstrated the effectiveness of the proposed strategy. Economic benefit for a building providing secondary frequency control to the grid varies with the availability of storage capacity in its HVAC system. Participation in the intraday market resulted in increased average benefit. Buildings providing AGC tracking service achieved higher comfort at reduced price. The economic benefit was sensitive to the electricity price, and hence to the physical location of the building within Switzerland.

\section{ACKNOWLEDGMENT}

We would like to thank Swissgrid Ltd. for providing us with all the relevant data for our simulation studies.

\section{REFERENCES}

[1] Y. G. Rebours, S. Member, D. S. Kirschen, and M. Trotignon, “A Survey of Frequency and Voltage Control Ancillary Services Part I : Technical Features," IEEE Transactions on Power Systems, vol. 22, no. 1, pp. 350-357, 2007.

[2] T. Borsche, A. Ulbig, M. Koller, and G. Andersson, "Power and energy capacity requirements of storages providing frequency control reserves," in Power and Energy Society General Meeting (PES), 2013 IEEE, July 2013, pp. 1-5.

[3] J. L. Sawin, F. Sverrisson, and W. Rickerson, "Renewables 2015 : Global Status Report," REN21, Tech. Rep., 2016.

[4] F. Oldewurtel, A. Ulbig, A. Parisio, G. Andersson, and M. Morari, "Reducing peak electricity demand in building climate control using real-time pricing and model predictive control," in Decision and Control (CDC), 2010 49th IEEE Conference on, Dec 2010, pp. 1927-1932.

[5] M. Maasoumy, C. Rosenberg, A. Sangiovanni-Vincentelli, and D. Callaway, "Model predictive control approach to online computation of demand-side flexibility of commercial buildings HVAC systems for supply following," in American Control Conference (ACC), 2014, June 2014, pp. 1082-1089.

[6] E. Vrettos, F. Oldewurtel, G. Andersson, and F. Zhu, "Robust Provision of Frequency Reserves by Office Building Aggregations," in Proceedings of the 19th IFAC World Congress, Cape Town, South Africa, 2014.

[7] M. Balandat, F. Oldewurtel, M. Chen, and C. Tomlin, "Contract design for frequency regulation by aggregations of commercial buildings," in 2014 52nd Annual Allerton Conference on Communication, Control, and Computing (Allerton), Sep. 2014, pp. 38-45.

[8] H. Hao, A. Kowli, Y. Lin, P. Barooah, and S. Meyn, “Ancillary service for the grid via control of commercial building hvac systems," in American Control Conference (ACC), 2013. IEEE, 2013, pp. 467-472.

[9] M. Maasoumy, "Flexibility of Commercial Building HVAC Fan as Ancillary Service for Smart Grid," IEEE Green Energy and Systems Conference (IGESC 2013), Nov. 2013.

[10] Y. Lin, P. Barooah, S. Meyn, and T. Middelkoop, "Experimental Evaluation of Frequency Regulation from Commercial Building HVAC system," IEEE Transaction on Smart Grid, vol. 6, no. no. 2, pp. 776783, March 2015.

[11] T. T. Gorecki, A. Bitlisliolu, G. Stathopoulos, and C. N. Jones, "Guaranteeing input tracking for constrained systems: Theory and application to demand response," in American Control Conference (ACC), 2015, July 2015, pp. 232-237.

[12] T. Borsche, A. Ulbig, M. Koller, and G. Andersson, "Power and energy capacity requirements of storages providing frequency control reserves," in IEEE PES General Meeting, Vancouver, 2013.

[13] I. Lymperopoulos, F. A. Qureshi, T. Nghiem, A. A. Khatir, and C. N. Jones, "Providing ancillary service with commercial buildings: the swiss perspective," in 9th (IFAC) Symposium on Advanced Control of Chemical Processes (ADCHEM), 2015. 


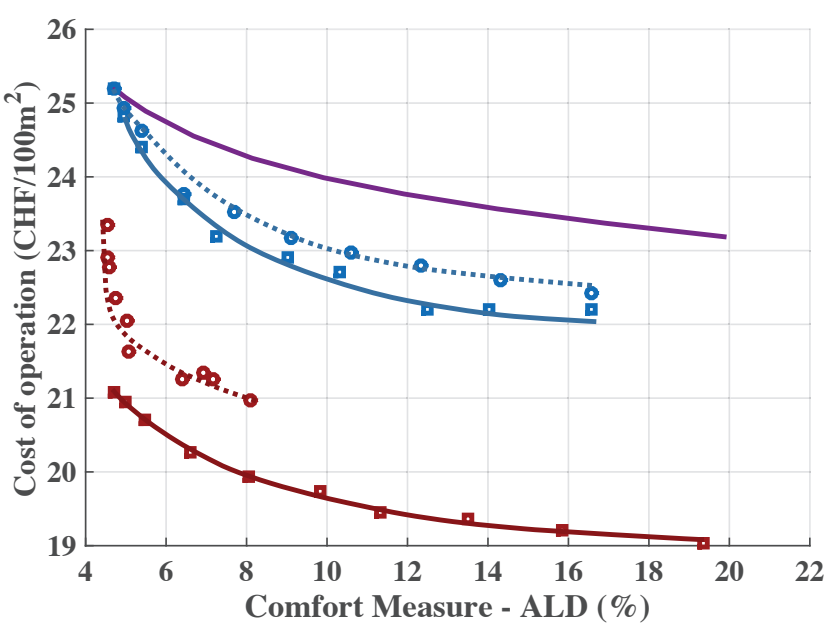

(a) Winter (Week 4)

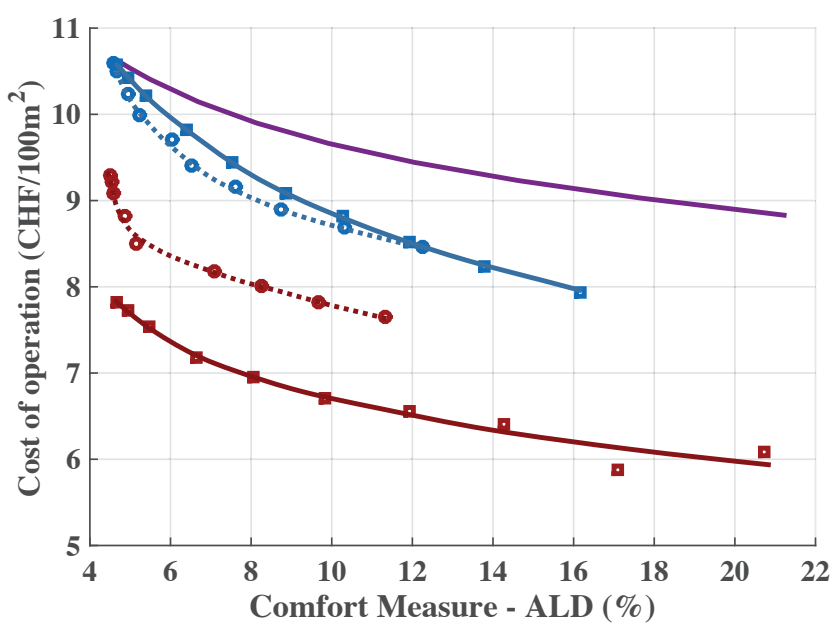

(b) Summer (Week 29)

Fig. 4: Cost of operation vs comfort: Solid purple: No AGC tracking without additional storage. Solid line: AGC Tracking Intraday case, Dotted line: AGC tracking - No Intraday. Red: Additional thermal storage, Blue: No additional thermal storage.

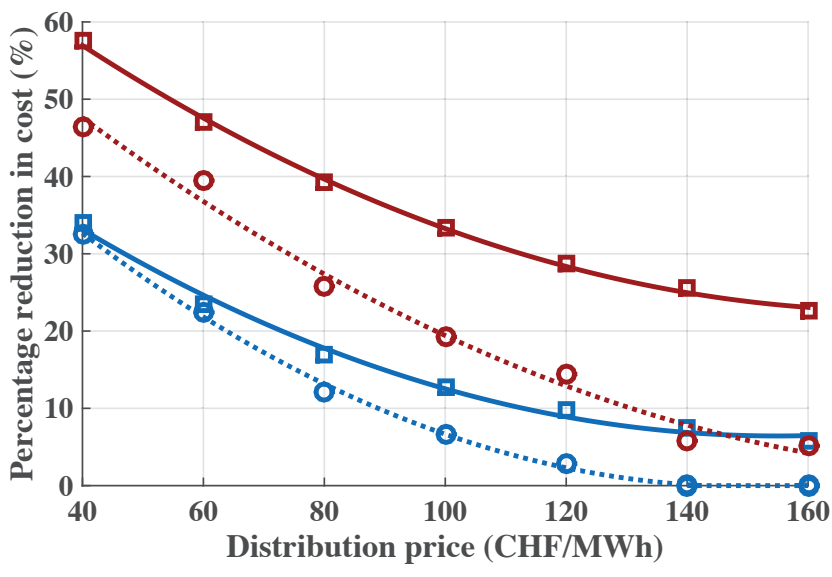

(a) Winter (Week 7)

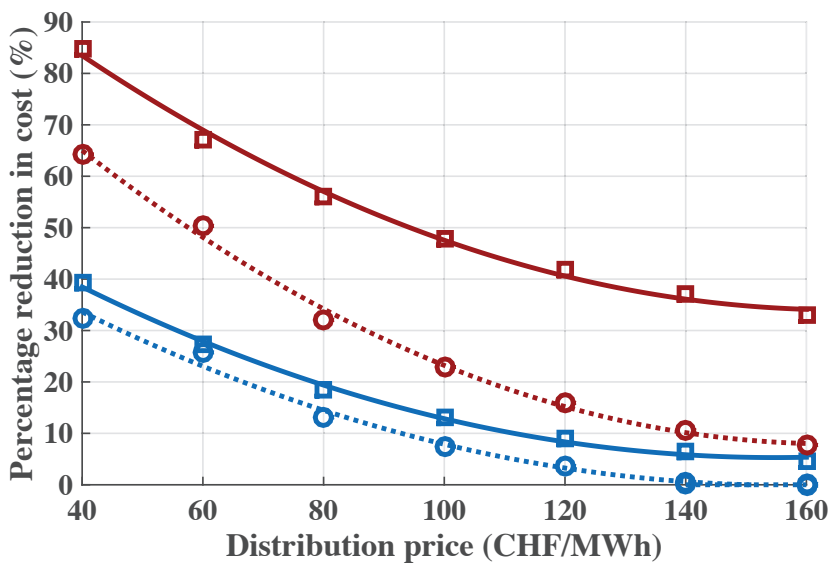

(b) Summer (Week 25)

Fig. 5: Percentage reduction in operating cost vs the distribution price of electricity: Solid line: AGC Tracking - Intraday case, Dotted line: AGC tracking - No Intraday case. Red: Additional thermal storage, Blue: No additional thermal storage.

[14] Y. G. Rebours, S. Member, D. S. Kirschen, and M. Trotignon, "A Survey of Frequency and Voltage Control Ancillary Services Part II : Economic Features," IEEE Transactions on Power Systems, vol. 22, no. 1, pp. 358366, 2007.

[15] SwissGrid, "Basic Principles of Ancillary Services Products." [Online]. Available: https://www.swissgrid.ch/dam/swissgrid/experts/ ancillary_services/Dokumente/D160111_AS-Products_V9R1_en.pdf

[16] T. Gorecki, F. Qureshi, and C. Jones, "Openbuild : An integrated simulation environment for building control," in Control Applications (CCA), 2015 IEEE Conference on, Sept 2015, pp. 1522-1527.

[17] A. Shapiro, D. Dentcheva et al., Lectures on stochastic programming: modeling and theory. SIAM, 2014, vol. 16.

[18] US Department of Energy: Office of Energy Efficiency and Renewable Energy, "Commercial Reference Buildings." [Online]. Available: http://energy.gov/eere/buildings/commercial-reference-buildings

[19] EpexSpot, "European Power Exchange." [Online]. Available: http: //www.epexspot.com/en/

[20] J. Lofberg, "Yalmip : a toolbox for modeling and optimization in matlab," in Computer Aided Control Systems Design, 2004 IEEE International Symposium on, Sept 2004, pp. 284-289.

[21] S. Carlucci, Thermal comfort assessment of buildings. Springer, 2013.

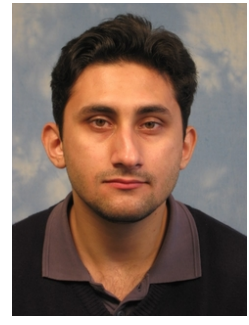

Faran A. Qureshi received the Bachelors degree in Electronic Engineering from the GIK Institute, Pakistan in 2009, and the Masters degree in Systems and Control from the Eindhoven University of Technology (TU/e), The Netherlands in 2012. In November 2012 he joined the group of Prof. Colin Jones in Automatic Control Laboratory at École Polytechnique Fédérale de Lausanne (EPFL), Switzerland. He is working in the areas of model predictive control applied to smart buildings, ancillary service provision, and grid integration. His research interests include economic MPC, stochastic, and robust optimization. 


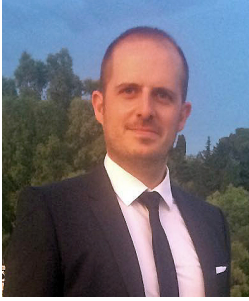

Ioannis Lymperopoulos has received his bachelor and masters degree from the University of Patras in Greece at the department of Electrical and Computer engineering. He has obtained his doctorate from the Automatic Control Lab at the Swiss Federal Institute of Technology (ETH Zurich) and has continued as a post-doc at the Swiss Federal Institute of Technology in Lausanne (EPFL). His interests include data-based learning and control, constrained-optimization and non-linear filtering. He has been working on the fields of renewable energy and Ancillary Services

provision.

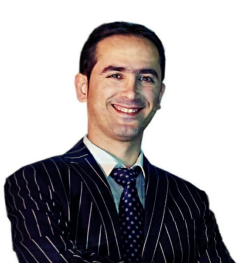

Ali Ahmadi-Khatir (S09M13) received the M.Sc. degree from Sharif University of Technology, Tehran, Iran, in 2005, and the Ph.D. degree from EPFL, Lausanne, Switzerland, in 2013. He is currently with Automatic Control Laboratory in EPFL as a Scientist. His research interests include electricity markets, power system operation, and optimization.

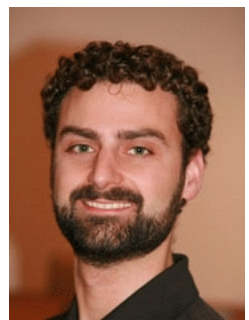

Colin N. Jones received the Bachelors degree in Electrical Engineering and the Masters degree in Mathematics from the University of British Columbia, Vancouver, BC, Canada, and the Ph.D. degree from the University of Cambridge, Cambridge, U.K., in 2005. He is an Assistant Professor in the Automatic Control Laboratory at the École Polytechnique Fédérale de Lausanne (EPFL), Lausanne, Switzerland. He was a Senior Researcher at the Automatic Control Laboratory of the Swiss Federal Institute of Technology Zurich until 2010. His current research interests are in the areas of high-speed predictive control and optimisation, as well as green energy generation, distribution and management. 